897

\section{USE OF TB-ELISPOT FOR DIAGNOSIS OF TUBERCULOSIS IN CHILDREN}

\author{
M.A. Hussain, BPAIIG \\ Paediatrics, East Surrey Hospital, Surrey \& \\ Sussex NHS Trust, Redhill, UK
}

Use of Interferon-y testing (TB-ELISPOT) for tuberculosis in children:

Aim of study: To look at usefulness of TB-Elispot for contact tracing for Tuberculosis in children in comparison with routine tuberculin skin test or mantouxBackground: UK On 22 March 2006, The National Institute for Health and Clinical Excellence (NICE) published their Guidelines for TB control in England and Wales. The NICE Guidelines recommend:

- Use IGRAs as the front line test for latent TB infection in preference to the tuberculin skin test (TST) where the skin test may be "less reliable" including all immunocompromised patients

- Use IGRAs as a secondary, confirmatory test in all cases when the TST is positive. The IGRA is used as a means of screening out TST false positives

- IGRAs also have a role to play in the diagnosis of TB disease especially in nonpulmonary TB and as a rule-out test in TB suspects

Methods: 40 children over a 15 month period had contact tracing done using the traditional tuberculin skin test or mantoux, followed by TB-Elispot where necessary as per national NICE guidance in UK.

Results: 10 cases out of 40 needed TB- Elispot; no negative ones where mantoux was positive. 1 case of TB-Elispot was positive even when mantoux was negative (done in view of close contact history and positive sputum)

Discussion: TB-Elispot appears to be a useful test in picking up both active and latent Tuberculosis in children and should be routinely used

\section{PANDEMIC INFLUENZA (H1N1) INFECTION IN PEDIATRIC AGE GROUP IN SOUTHERN IRAN}

A. Moattari ${ }^{1}$, A. Emami ${ }^{1}$, K. Bagheri Lankarani ${ }^{2}$

${ }^{1}$ Virology, ${ }^{2}$ Shiraz Medical University, Shiraz, Iran

Background: A novel strain of H1N1 Influenza (A/ California/7/2009) has been prevalent since April 2009. Epidemiological and clinical studies showed that children at high risk for severe infection.

Objective: to determined the prevalence of new H1N1 in children with fever, cough,sore throat, bronchiolitis, pneumonia and wheezing symptoms.

Methods: pharyngeal swabs were taken from 450 children aged (1-60 months) with respiratory tract symptom between June 2009 to April 2010. The specimens were tested using real time reverse transcription-PCR.

Results: out of 450 samples 130 were tested positive for pandemic H1N1 infection.

Conclusion: According to our results $29.1 \%$ of respiratory infection in children in the south of Iran was due to new H1N1 during the foregoing pandemic. Our analysis revealed no significant correlation between males and females.

Key words: new H1N1, Iran, pediatrics influenza

899

\section{PREVALENCE OF HUMAN METAPNEUMOVIRUS (HMPV) IN CHILDREN WITH RESPIRATORY DISEASE SYMPTOMS - SHIRAZ-IRAN}

\author{
A. Emami ${ }^{1}$, A. Moattari ${ }^{2}$, M. Moghadami ${ }^{3}$ \\ ${ }^{1}$ Shiraz Medical School, ${ }^{2}$ Virology, Shirtaz Medical \\ School, ${ }^{3}$ Shirtaz Medical University, Shiraz, Iran
}

Background: Human metapneumovirus (hMPV) is one of the main causes of viral lower and upper respiratory tract infections which was the first isolated in 2001 from children with acute respiratory tract infections. hMPV have been classified in the Pneumovirinae subfamily within the Paramyxoviridae family. Since then, increasingly world wide investigators have been reported that hMPV is a leading cause of lower respiratory tract infections in children.

Objective: In this study we tried to determine the prevalence of human pneumovirus in hospitalized 